\title{
Characterization of virulent Newcastle disease viruses from vaccinated chicken flocks in Eastern China
}

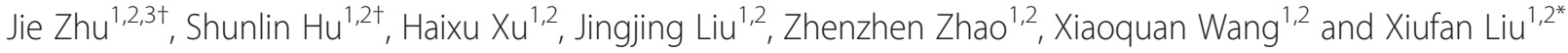

\begin{abstract}
Background: Newcastle disease (ND) is one of the most contagious and devastating diseases to poultry in the world. The causative agents are virulent strains of Newcastle disease virus (NDV), which belong to the genus Avulavirus, sub-family Paramyxoviridae, family Paramyxovirinae. Knowing the genomic and antigenic characteristics of virulent NDVs might contribute to ND control in China.

Results: The results showed that all of the virulent strains belonged sub-genotype VIld shared the same cleavage site ${ }^{112}$ RRQKR/F ${ }^{117}$ in the fusion protein. At least $69 \%$ (38 of 55) of the NDV strains possessed E347K variation in the hemagglutinin-neuraminidase protein. The cross-neutralization tests confirmed that the strains harboring $347 \mathrm{~K}$ showed lower antigenic relatedness with LaSota. Furthermore, the immune-challenge experiment indicated that LaSota could not provide complete protection against infection with the E347K variant NDVs as the vaccinated birds were still able to be infected and shed virulent challenge viruses.

Conclusions: Currently, sub-genotype VIId NDVs are the prevalent virulent strains circulating among vaccinated chicken flocks in Eastern China. Our findings indicated that the E347K variation in HN gene would expand the antigenic difference with LaSota, which may be responsible for the increasing isolation rate of these strains from vaccinated chickens.
\end{abstract}

Keywords: Virulent Newcastle disease virus, Sub-genotype VIId, E347K, Variation, Antigenic difference

\section{Background}

Newcastle disease (ND) is one of the most contagious and devastating diseases to poultry in the world [1]. The causative agents are virulent strains of Newcastle disease virus (NDV), which belong to the genus Avulavirus, sub-family Paramyxoviridae, family Paramyxovirinae [2, 3]. The viral RNA genome is approximately $15 \mathrm{~kb}$ in length and encodes six major virus proteins: the nucleocapsid protein $(\mathrm{NP})$, phosphoprotein $(\mathrm{P})$, matrix protein $(\mathrm{M})$, fusion protein $(\mathrm{F})$, hemagglutinin-neuraminidase $(\mathrm{HN})$ and large polymerase protein $(\mathrm{L})$. In addition, the $\mathrm{HN}$ protein is an important multifunctional surface

\footnotetext{
* Correspondence: xfliu@yzu.edu.cn

${ }^{\dagger}$ Equal contributors

${ }^{1}$ Animal Infectious Disease Laboratory, College of Veterinary Medicine,

Yangzhou University, 12 East Wenhui Road, Yangzhou, Jiangsu 225009, China

${ }^{2}$ Jiangsu Co-innovation Center for Prevention and Control of Important

Animal Infectious Diseases and Zoonoses, Yangzhou University, Yangzhou

225009, China

Full list of author information is available at the end of the article
}

glycoprotein that consists of a cytoplasmic domain, a transmembrane region, a stalk region and a globular head, in which the receptor-binding site, the site responsible for neuraminidase activity and all of the antigenic sites reside [4]. It has been confirmed that there are seven overlapping antigenic sites in the HN protein, whereas residues 345 to 353 constitute the only linear epitope identified in the HN gene, which is susceptible to immune pressure to generate antigenic variation [5]. Researchers have recently confirmed that the variation in the linear epitope in the HN protein would intensify the antigenic difference [6]. Previously, seven neutralizing epitopes positioned at residues $72,74,75,78,79$, 157 to 171, and 343 of the $F$ protein have been identified.

The strains of NDV can be divided into two distinct clades: class I and class II, and based on the older classification system, both clades could be divided into 9 genotypes, and the class II clade can be divided into fifteen 
genotypes when based on the new classification system, while the class I clade can be divided into three subgenotypes. To date, a strict vaccination policy has been implemented in China and the vaccine strain LaSota which belonged to genotype II has been widely used in China for over 40 years. However, infections of genotype VIId NDVs have still frequently occurred in China and other Asian countries [7-13]. Meanwhile, there are also some virulent genotypes (VIb, IX) circulating among avian species in China $[14,15]$.

To elucidate the circulation of virulent NDVs among chickens in Eastern China, 55 virulent NDVs isolated from chicken flocks, including broilers and layers, from 2011 to 2013 were phylogenetically characterized.

\section{Results}

Isolation and identification of NDVs from clinical samples Fifty-five NDVs from clinical samples of different chicken flocks in Eastern China were isolated from 2011 to 2013, identified and plaque-purified, and the details of the NDV isolates are shown in Table 1.

\section{Phylogenetic analysis}

The phylogenetic analysis of the 55 NDVs based on the whole $\mathrm{F}$ gene sequences showed that all strains were clustered into sub-genotype VIId and they could be divided into two separate groups, namely VIId1 $(n=3)$ and VIId2 $(n=52)$, as previously described (Fig. 1) [7]. All of the strains isolated in 2012-2013 belonged to VIId2, and only three of the twenty one strains isolated in 2011 belonged to VIId1. All of the NDV isolates possessed the virulent $\mathrm{F}$ protein cleavage site motif ${ }^{112} \mathrm{RRQKR} / \mathrm{F}^{117}$. And over $70 \%(39 / 55)$ of the isolates shared $\mathrm{K} 78 \mathrm{R}$ variation on the $\mathrm{F}$ gene.

Additionally, a phylogenetic analysis was also performed based on the whole HN gene (Fig. 2). As determined from the phylogenetic tree based on the $\mathrm{HN}$ gene, all of the NDV isolates showed a distribution similar to that obtained from the tree based on the F genes: three in VIId1 and 52 in VIId2. Three residues, namely T102, A118 and T443, were unique for VIId1, whereas I102, E118 and M443 were characteristic residues of VIId2. All of the NDV isolates shared $96.3 \%$ to $100 \%$ nucleotide identity and $96.2 \%$ to $99.8 \%$ amino acid identity. Only two isolates (JS-08-11-Ch and JS-10-11$\mathrm{Ch}$ ) shared the E347G variation. More than $42.8 \%$ of the isolates in $2011(9 / 21)$ exhibited the E347K variation in $\mathrm{HN}$, whereas $81.5 \%$ (22 of 27 ) of those isolated in 2012 possessed this variation. The latter percentage is nearly two-fold higher than that found in 2011, and $100 \%$ (7 of 7) of the strains isolated in 2013 possessed this variation. In addition, the layer-origin stains isolated in 2011, 2012 and 2013 shared $67 \%$ (6/9), $75 \%(6 / 8)$ and $100 \%(3 / 3)$ of the E347K variation in the $\mathrm{HN}$ gene, respectively. Furthermore, we found that all of the strains harboring $347 \mathrm{~K}$ on $\mathrm{HN}$ also shared a G362A mutation. However, the role of this mutation has not been well elucidated. The 13 cysteine residues were conserved as described previously, and with the exception of the loss of a glycosylation site at 508 of JS-02-11, all of the other NDVs possessed six well-identified glycosylation sites.

\section{Pathogenicity tests}

As is shown in Table 2, all the ten isolates were highly virulent, which were consistent with the tyipical virulent motif ${ }^{112} R R Q K R F^{117}$ at the $F$ cleavage sites.

\section{Cross hemagglutination inhibition (HI) test and virus neutralization test}

The HI titers of the anti-LaSota serum for the ten isolates were 4-8-fold lower than that for the homologous strain LaSota, displaying the significance of the antigenic difference between the NDV isolates and the vaccine strain LaSota. As determined from the cross HI test, the $R$ values between the predominant strains and LaSota were all lower than 0.5 , indicating the existence of a significant antigenic difference between the strains. Meanwhile the $R$ values between the E347K-variant strains and LaSota were lower than that found for the 347E/G strains, which indicate that the E/G347K variation would expand the antigenic difference with the vaccine strain. And the significant antigenic difference was also confirmed by the $\mathrm{R}$ value obtained from cross-neutralization test between LaSota and the predominant strains (Table 3).

\section{Test of the protective efficacy of the LaSota strain against the variant strains}

As is shown in Table 4, none of the birds vaccinated with the LaSota vaccine showed clinical signs post challenge with JS-22-11-Ch or JS-14-12-Ch. In contrast, $100 \%$ of the unvaccinated birds challenged with JS-2211-Ch or JS-14-12-Ch died within five days post challenge. All of the unchallenged birds remained normal throughout the experiment.

As is shown in Table 5, tracheal and cloacal swabs were collected from the birds at days 3, 5, and 7 post challenge and subjected to virus isolation tests. The virus isolation rate from the tracheal swabs was $100 \%(5 / 5)$ at day 3 post challenge in both the control-JS22 and control-JS14 groups, whereas the isolation rates for the La-JS22 and La-JS14 groups were $70 \%(7 / 10)$ and $100 \%$ (10/10), respectively. And the virus isolation rate from the tracheal swabs was $50 \%(5 / 10)$ and $70 \%(7 / 10)$ at day 5 post challenge in La-JS22 and La-JS14 groups, whereas the isolation rates at day 7 post challenge were $10 \%(1 / 10)$ and $30 \%(3 / 10)$, respectively. 
Table 1 NDVs characterized in this study

\begin{tabular}{|c|c|c|c|c|c|c|c|c|c|c|c|}
\hline NDV isolates & $\begin{array}{l}\text { Year of } \\
\text { isolation }\end{array}$ & Host & F gene & $\begin{array}{l}345-353 \\
\text { Residues of HN }\end{array}$ & HN gene & NDV isolates & $\begin{array}{l}\text { Year of } \\
\text { isolation }\end{array}$ & Host & F gene & $\begin{array}{l}\text { 345-353 } \\
\text { Residues of HN }\end{array}$ & HN gene \\
\hline JS-01-11-Ch & 2011 & Broiler & JQ013855 & PDEQDYQIR & JQ013835 & JS-09-12-Ch & 2012 & Layer & KJ184579 & PDKQDYQIR & KJ184625 \\
\hline JS-03-11-Ch & 2011 & Broiler & JQ013857 & PDEQDYQIR & JQ013838 & JS-10-12-Ch & 2012 & Layer & KJ184580 & PDKQDYQIR & KJ184626 \\
\hline JS-04-11-Ch & 2011 & Layer & JQ013858 & PDEQDYQIR & KJ184630 & JS-11-12-Ch & 2012 & Layer & KJ184581 & PDKQDYQIR & KJ184627 \\
\hline JS-05-11-Ch & 2011 & Layer & JQ013859 & PDEQDYQIR & KJ184631 & JS-12-12-Ch & 2012 & Broiler & KJ184582 & PDKQDYQIR & KJ184601 \\
\hline JS-06-11-Ch & 2011 & Layer & JQ013860 & PDKQDYQIR & JQ013839 & JS-13-12-Ch & 2012 & Broiler & KJ184583 & PDKQDYQIR & KJ184602 \\
\hline JS-07-11-Ch & 2011 & Broiler & JQ013861 & PDKQDYQIR & KJ184632 & JS-14-12-Ch & 2012 & Broiler & KJ184584 & PDKQDYQIR & KJ184603 \\
\hline JS-08-11-Ch & 2011 & Broiler & JQ013862 & PDGQDYQIR & KJ184633 & $\mathrm{AH}-02-12-\mathrm{Ch}$ & 2012 & Layer & KJ184597 & PDKQDYQIR & KJ184616 \\
\hline JS-09-11-Ch & 2011 & Broiler & JQ013863 & PDEQDYQIR & KJ184634 & HeN-01-12-Ch & 2012 & Layer & KJ184598 & PDKQDYQIR & KJ184617 \\
\hline JS-10-11-Ch & 2011 & Layer & JQ013864 & PDGQDYQIR & JQ013840 & SD-01-12-Ch & 2012 & Broiler & KJ184594 & PDKQDYQIR & KJ184613 \\
\hline JS-11-11-Ch & 2011 & Layer & JQ013865 & PDKQDYQIR & JQ013841 & SD-02-12-Ch & 2012 & Broiler & KJ184595 & PDKQDYQIR & KJ184614 \\
\hline JS-12-11-Ch & 2011 & Broiler & JQ013866 & PDEQDYQIR & JQ013842 & SD-03-12-Ch & 2012 & Broiler & KJ184596 & PDKQDYQIR & KJ184615 \\
\hline JS-13-11-Ch & 2011 & Broiler & JQ013867 & PDEQDYQIR & JQ013843 & JS-16-12-Ch & 2012 & Broiler & KJ184585 & PDKQDYQIR & KJ184604 \\
\hline JS-14-11-Ch & 2011 & Broiler & JQ013868 & PDKQDYQIR & JQ013852 & JS-17-12-Ch & 2012 & Broiler & KJ184586 & PDKQDYQIR & KJ184605 \\
\hline JS-15-11-Ch & 2011 & Broiler & JQ013869 & PDEQDYQIR & JQ013844 & JS-18-12- Ch & 2012 & Broiler & KJ184587 & PDEQDYQIR & KJ184606 \\
\hline JS-16-11-Ch & 2011 & Broiler & JQ013870 & PDKQDYQIR & JQ013845 & JS-19-12-Ch & 2012 & Broiler & KJ184588 & PDKQDYQIR & KJ184607 \\
\hline JS-17-11-Ch & 2011 & Layer & JQ013871 & PDKQDYQIR & JQ013846 & JS-20-12-Ch & 2012 & Broiler & KJ184589 & PDKQDYQIR & KJ184608 \\
\hline JS-19-11-Ch & 2011 & Layer & JQ013873 & PDKQDYQIR & JQ013847 & JS-21-12-Ch & 2012 & Broiler & KJ184590 & PDKQDYQIR & KJ184609 \\
\hline JS-20-11-Ch & 2011 & Broiler & JQ013874 & PDEQDYQIR & JQ013848 & JS-22-12-Ch & 2012 & Broiler & KJ184591 & PDEQDYQIR & KJ184610 \\
\hline JS-21-11-Ch & 2011 & Broiler & JQ013875 & PDEQDYQIR & JQ013851 & JS-23-12-Ch & 2012 & Broiler & KJ184592 & PDKQDYQIR & KJ184611 \\
\hline SD-01-11-Ch & 2011 & Layer & JQ013877 & PDKQDYQIR & JQ013850 & JS-24-12-Ch & 2012 & Broiler & KJ184593 & PDKQDYQIR & KJ184612 \\
\hline SD-02-11-Ch & 2011 & Layer & JQ013878 & PDKQDYQIR & JQ013849 & JS-21-13-Ch & 2013 & Layer & KP064014 & PDKQDYQIR & KP064023 \\
\hline JS-02-12-Ch & 2012 & Layer & KJ184599 & PDKQDYQIR & KJ184618 & JS-22-13-Ch & 2013 & Broiler & KP064015 & PDKQDYQIR & KP064024 \\
\hline JS-03-12-Ch & 2012 & Broiler & KJ184600 & PDEQDYQIR & KJ184619 & JS-23-13-Ch & 2013 & Broiler & KP064016 & PDKQDYQIR & KP064025 \\
\hline JS-04-12-Ch & 2012 & Broiler & KJ184574 & PDEQDYQIR & KJ184620 & JS-24-13-Ch & 2013 & Layer & KP064017 & PDKQDYQIR & KP064027 \\
\hline JS-05-12-Ch & 2012 & Broiler & KJ184575 & PDEQDYQIR & KJ184621 & JS-27-13-Ch & 2013 & Layer & KP064018 & PDKQDYQIR & KP064021 \\
\hline JS-06-12-Ch & 2012 & Layer & KJ184576 & PDKQDYQIR & KJ184622 & JS-30-13-Ch & 2013 & Broiler & KP064020 & PDKQDYQIR & KP064026 \\
\hline JS-07-12-Ch & 2012 & Layer & KJ184577 & PDKQDYQIR & KJ184623 & SD-25-13-Ch & 2013 & Broiler & KP064019 & PDKQDYQIR & KP064022 \\
\hline JS-08-12-Ch & 2012 & Broiler & KJ184578 & PDKQDYQIR & KJ184624 & & & & & & \\
\hline
\end{tabular}

\section{Discussion}

The genotype VII NDVs have become the most prevalent strains in China since the 1990s, and the subgenotype VIId NDVs are mainly responsible for the present ND epizootic in China $[7,13,16]$. In this study, virulent NDVs from the vaccinated chicken flocks were characterized as sub-genotype VIId and could be clustered into sub-genotype VIId1 and VIId2 based on the phylogenetic trees based on both $\mathrm{F}$ and $\mathrm{HN}$ genes [7]. T102, A118, and T443 of the HN gene were unique residue substitutions found in the VIId1 NDVs, and I102, $\mathrm{E} 118$, and M443 of the HN gene were characteristic residues of the VIId2 NDVs. In this study, we found that sub-genotype VIId2 remains the predominant genotype VII NDVs from 2011 to 2013, and all of the NDVs isolated from 2012 to 2013 were located in this clade.
The HN protein of NDV plays an important role in immune protection against virus infection, and the variation of the antigenic epitope would expand the antigenic difference. And analysis of the role of site 347 in antigenicity has confirmed that the E347K variation would intensify the antigenic difference and may increase the risk of vaccine breakdown $[17,18]$. The isolation rate of strains with the E347K variation is increasing yearly, as previous reported. More than $36 \%$ of the virus strains $(20 / 55)$ were isolated in commercial layer flocks, which have resulted in losses in egg production. Cho et al. [18] found that the Korean variation strains have also caused losses in egg production. Moreover, more than $75 \%(15 / 20)$ of the NDV strains isolated from layer flocks were the E347K-variation strains. The high isolation rate indicates that the E347K-variation strains can 


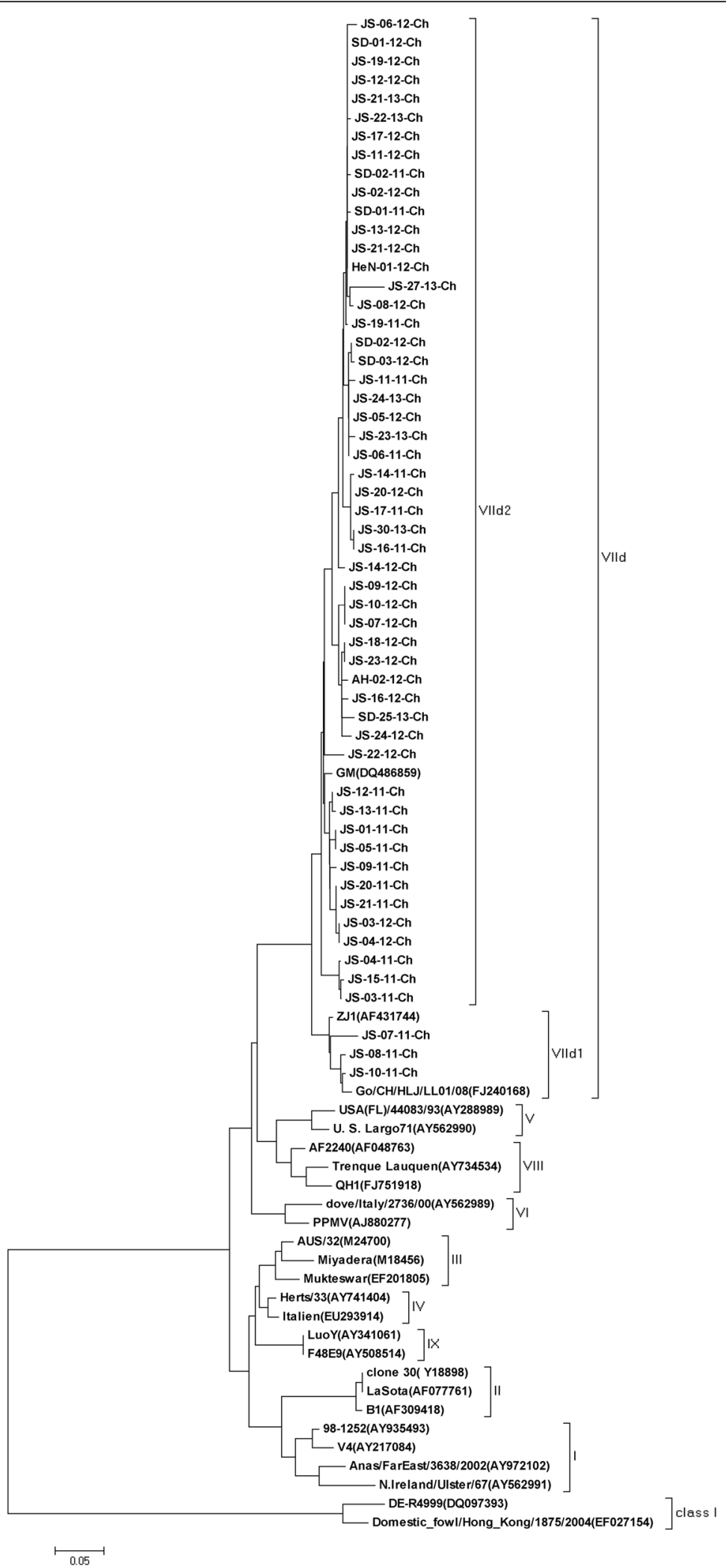

Fig. 1 Phylogenetic tree of 55 sub-genotype VIId NDV strains based on the whole region of the F gene 


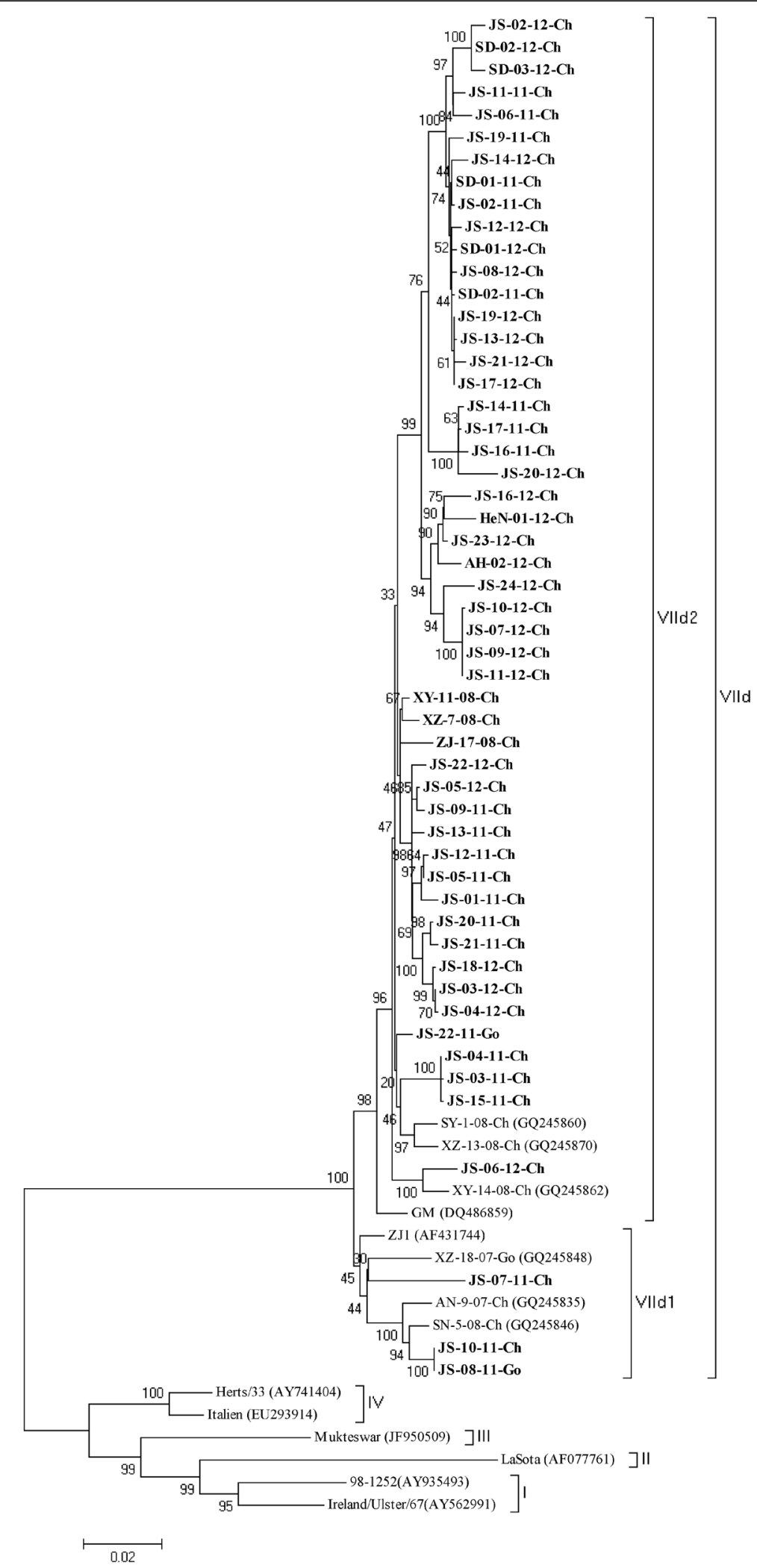

Fig. 2 Phylogenetic tree of 55 sub-genotype VIId NDV strains based on the complete HN gene 
Table 2 Coefficients of antigenic similarity (R) between NDV isolates and LaSota strain

\begin{tabular}{|c|c|c|}
\hline Strains & $R$ value $^{c}$ & 345-353 Residues of HN \\
\hline SD-01-11-Ch & $0.04^{a} 0.12^{b}$ & PDKQRDYQIR \\
\hline JS-07-11-Ch & $0.12^{a} 0.12^{b}$ & PDKQDYQIR \\
\hline JS-14-12-Ch & $0.06^{\mathrm{a}} 0.12^{\mathrm{b}}$ & PDKQDYQIR \\
\hline $\mathrm{HeN}-01-12-\mathrm{Ch}$ & $0.12^{\mathrm{a}} 0.12^{\mathrm{b}}$ & PDKQDYQIR \\
\hline JS-27-13-Ch & $0.17^{\mathrm{a}} 0.25^{\mathrm{b}}$ & PDKQDYQIR \\
\hline JS-08-11-Ch & $0.35^{\mathrm{a}} 0.35^{\mathrm{b}}$ & PDGQDYQIR \\
\hline JS-15-11-Ch & $0.35^{\mathrm{a}} 0.5^{\mathrm{b}}$ & PDEQDYQIR \\
\hline JS-05-12-Ch & $0.35^{\mathrm{a}} 0.35^{\mathrm{b}}$ & PDEQDDYQIR \\
\hline JS-18-12-Ch & $0.25^{\mathrm{a}} 0.35^{\mathrm{b}}$ & PDEQDYQIR \\
\hline JS-22-12-Ch & $0.35^{a} 0.35^{b}$ & PDEQDYQIR \\
\hline
\end{tabular}

${ }^{a}$ Chicken embryo cross-neutralization test

${ }^{\mathrm{b}}$ Cross-hemagglutination inhibition test

${ }^{\mathrm{c}} 0.67 \leq R \leq 1.5$, indicates no significant antigenic difference between the two viruses

$0.5 \leq R \leq 0.67$ indicates a minor difference between the two viruses

$R<0.5$ indicates a major difference between the two virus strains

infect chickens under immune pressure because the layers were all well-vaccinated in China. Both site 347 and site 362 locate around the receptor-binding pocket, and we found of E347K, G362A co-variant strains, whereas Cho et al. also identified two strains with the E347K and G362R co-variant ones. However, the role of the co-variation remains unknown [6].

At present, LaSota is widely and frequently used to prevent NDV infection in the poultry industries in China; however, virulent NDV infections continue to occur in the well-vaccinated chicken flocks [12, 19]. Moreover, both cross $\mathrm{HI}$ and cross-neutralization tests were performed, and the $\mathrm{R}$ values of all of the strains were lower than 0.5 . However, the $\mathrm{R}$ values between the E347K-variant strains and LaSota were lower than that found for the $347 \mathrm{E} / \mathrm{G}$ strains, which indicate that the E347K variation would expand the antigenic difference

Table 3 Pathogenicity tests of the isolates

\begin{tabular}{lll}
\hline Strains & $\mid \mathrm{ICP}^{\mathrm{a}}$ & $\mathrm{MDT}^{\mathrm{b}}(\mathrm{h})$ \\
\hline SD-01-11-Ch & 1.84 & 48 \\
JS-07-11-Ch & 1.86 & 46.8 \\
JS-14-12-Ch & 1.94 & 43.8 \\
HeN-01-12-Ch & 1.82 & 52 \\
JS-27-13-Ch & 1.86 & 46 \\
JS-08-11-Ch & 1.94 & 46 \\
JS-15-11-Ch & 1.92 & 48 \\
JS-05-12-Ch & 1.96 & 49.2 \\
JS-18-12-Ch & 1.84 & 48 \\
JS-22-12-Ch & 1.86 & 46 \\
\hline
\end{tabular}

${ }^{\mathrm{a} M D T}=$ mean death time

${ }^{\mathrm{b}} \mathrm{ICPI}=$ intracerebral pathogenicity index with the vaccine strain. Therefore, the results of the cross-neutralization and cross $\mathrm{HI}$ experiments may also be consistent with the higher isolation rate of the E347K-variant strains, which demonstrated significant antigenic differences between the vaccine strain and the predominant variant strains should be responsible for the outbreaks of ND in China.

In addition, the protection efficiency of LaSota against the E347K variation strains in China is not wellelucidated. In this study, the vaccinated SPF chickens were fully protected against morbidity and mortality, but virus shedding was not stopped. Our data suggest that the LaSota inactivated vaccine cannot protect chickens from virus shedding when infected with the variant NDVs, and the variant strain challenged group showed higher virus isoation rate which may be one of the reasons for the circulation of sub-genotype VIId variation strains in vaccinated chicken flocks.

In conclusion, our findings indicate that the subgenotype VIId NDVs with E347K variation in the HN protein are predominant in eastern China and that this variation would intensify the antigenic difference with the vaccine strains. Moreover, the vaccine strain LaSota has been used for more than 40 years and can hardly protect chickens effectively from virus shedding when infected with the circulation strains. Therefore, new NDV vaccines closely related to the prevalent genotype VIId viruses should be developed to control ND in China.

\section{Conclusions}

Currently, sub-genotype VIId NDVs are the prevalent virulent strains circulating among vaccinated chicken flocks in Eastern China. Our findings indicate that the E347K variation in $\mathrm{HN}$ protein would expand the antigenic difference with LaSota, which may be responsible for the increasing isolation rate of these strains from vaccinated chickens.

\section{Methods}

\section{Virus isolation and antiserum preparation}

Fifty-five virulent NDVs were isolated from 190 clinical samples from different flocks in Eastern China from 2011 to 2013 under the permission of the flock owners. All of the viruses were plaque-purified three times on primary chicken embryo fibroblasts (CEF) and proliferated in 9-10-day-old specific-pathogen-free (SPF) chicken embryos [20]. The virus stocks grown in allantoic fluids were stored at $-70{ }^{\circ} \mathrm{C}$ until use.

SPF chickens (five in each group) were vaccinated with inactivated, oil-emulsion derived from LaSota and the strains listed in Table 2. The serum for the five birds vaccinated with the same vaccine were pooled together 
Table 4 Protection of vaccinated SPF chickens with inactivated oil-emulsion vaccines against challenge with the predominant variant NDVs

\begin{tabular}{|c|c|c|c|c|c|c|}
\hline \multirow[t]{2}{*}{ Vaccine-Challenge $^{a}$} & \multirow{2}{*}{$\begin{array}{l}\text { HI titer }(\log 2) \\
\text { Prior to } \\
\text { challenge }\end{array}$} & \multicolumn{3}{|l|}{ Challenge } & \multicolumn{2}{|l|}{ Protection } \\
\hline & & Strain & Route & NO. birds & Clinical signs & Mortality \\
\hline La-JS22 & $6.3 \pm 1.8$ & JS-01-11-Ch & $\mathrm{ED} / \mathrm{IN}$ & 10 & $0 / 10$ & $0 \%(0 / 10)$ \\
\hline La-JS14 & $6.3 \pm 1.8$ & JS-14-12-Ch & $\mathrm{ED} / \mathrm{IN}$ & 10 & $0 / 10$ & $0 \%(0 / 10)$ \\
\hline Control-JS22 & 0 & JS-01-11-Ch & $\mathrm{ED} / \mathrm{IN}$ & 5 & $5 / 5$ & $100 \%(5 / 5)$ \\
\hline Control -JS14 & 0 & JS-14-12-Ch & $\mathrm{ED} / \mathrm{IN}$ & 5 & $5 / 5$ & $100 \%(5 / 5)$ \\
\hline La-PBS & $6.3 \pm 1.8$ & PBS & $\mathrm{ED} / \mathrm{IN}$ & 5 & $0 / 10$ & $0 \%(0 / 5)$ \\
\hline Control-PBS & 0 & PBS & $\mathrm{ED} / \mathrm{IN}$ & 5 & $0 / 10$ & $0 \%(0 / 5)$ \\
\hline
\end{tabular}

${ }^{a}$ La-JS22 and Control-JS22 = Challenge with $10^{6}$ EID $_{50}$ JS-22-11-Ch; La-JS14 and Control-JS14 = Challenge with $10^{6}$ EID $_{50}$ JS-14-12-Ch

at three weeks post-vaccination and stored at $-70{ }^{\circ} \mathrm{C}$ until use.

All of the SPF chicken embryos and the SPF chickens used in this study were bought from Beijing Merial Vital Laboratory Technology Co., LTD.

\section{Viral RNA purification, RT-PCR, and sequencing of the $\mathrm{F}$ and $\mathrm{HN}$ genes}

Virus preparation, viral RNA extraction and the initial RT reaction were performed as described previously [21]. Based on the published $\mathrm{F}$ and $\mathrm{HN}$ gene sequences in the GenBank database, we designed two pairs of primers (Table 6) for amplifying the F and HN genes of the virulent NDV isolates. And we also designed a pair of primers based one the same region of all NDV F genes which could be used for NDV identification. PCR products possessing the expected length were purified and sequenced by Sangon (Shanghai, China).

\section{Sequence analysis}

Nucleotide sequence editing, analysis and prediction of the amino acid sequences for both the $\mathrm{F}$ and HN proteins and alignments were conducted using the Clustal W multiple alignment method in the MegAlign program of the Lasergene package (DNASTAR Inc., Madison, WI, USA). Phylogenetic trees based on the F and HN genes were constructed using the MEGA 5 program (Version 5.2) with the maximum likelihood method algorithm [22]. In addition to the 55 strains collected in this study, 31 previously reported $\mathrm{F}$ and $\mathrm{HN}$ gene sequences representing different genotypes were included for comparison, and the accession numbers of each of these NDVs are shown in the phylogenetic trees $[7,16,21]$.

\section{Pathogenicity tests}

The intracerebral pathogenicity index in 1-day-old chicks and the mean death time in 9 to 11-day-old SPF chicken embryos were determined for some of the isolates in this study as Liu did previously [7].

\section{Cross hemagglutination inhibition (HI) test and virus neutralization test}

To measure the antigenic difference between the vaccine strain LaSota and the isolated strains, we selected ten strains (One harboring 347G, four harboring 347E, and five harboring $347 \mathrm{~K}$ ) and performed cross $\mathrm{HI}$ and virus neutralization test as describe by $\mathrm{Cho}$ and $\mathrm{Li}[8,23]$. Briefly, virus-serum mixtures were inoculated into the allantoic cavity of 10-day-old SPF chicken embryos $0.2 \mathrm{~mL}$ per sample. At the same time, blank controls and no-serum control samples were prepared by inoculating with an equal volume of PBS or virus. Eight days after incubation, the virus present in the chorioallantoic sac was titrated by calculating the $50 \%$ virus neutralization endpoint. Virus neutralizing activity was determined relative to the no-serum control. The antigenic relatedness of the predominant strains and LaSota strain was expressed using the $\mathrm{R}$ value, as described by Archetti and Horsfall [24]. The following formula was used: $\mathrm{R}=\sqrt{r 1 * r 2}$, where $\mathrm{r} 1$ is the titer of strain $\mathrm{A}$ with antiserum $\mathrm{B}$ divided by the titer of strain $\mathrm{A}$ with antiserum A, and $\mathrm{r} 2$ is the titer of strain B with antiserum A divided by the titer of strain $B$ with antiserum B. A result of $0.67 \leq R \leq 1.5$ indicates no significant antigenic difference between the two viruses, whereas $0.5 \leq$ $R \leq 0.67$ indicates a minor antigenic difference between

Table 5 Frequency of isolation of challenge virus from SPF chickens

\begin{tabular}{|c|c|c|c|c|c|c|}
\hline \multirow[t]{3}{*}{ Group } & \multicolumn{6}{|c|}{ Samples (positive/total) } \\
\hline & \multicolumn{2}{|c|}{ Day 3 pc } & \multicolumn{2}{|c|}{ Day 5 pc } & \multicolumn{2}{|c|}{ Day 7 pc } \\
\hline & OS & CS & OS & CS & OS & CS \\
\hline La-JS22 & $7 / 10$ & $2 / 10$ & $5 / 10$ & $6 / 10$ & $1 / 10$ & $2 / 10$ \\
\hline La-JS14 & $10 / 10$ & $5 / 10$ & $7 / 10$ & $7 / 10$ & $3 / 10$ & $4 / 10$ \\
\hline Control-SD & $5 / 5$ & $5 / 5$ & - & - & - & - \\
\hline Control-JS & $5 / 5$ & $5 / 5$ & - & - & - & - \\
\hline
\end{tabular}

-the birds were all dead within the day $5 \mathrm{pc}$

OS = oral swabs; $\mathrm{CS}=$ cloacal swabs 
Table 6 Primers used in the study

\begin{tabular}{llll}
\hline Fragment designation & Primer sequence $\left(5^{\prime}-3^{\prime}\right)$ & Position & Expected size (bp) \\
\hline FTY1 & CgTAgA AAA AACACgggTAgAAgA & $4494-4517$ & 958 \\
FTY2 & CAggTAggTRgCACgCATATT ATT & $5429-5452$ & 1662 \\
F1 & ATGGGCTCCAAACCTTCTA & $4550-4568$ & $6195-6212$ \\
F2 & TCCTGTGGTGGCTCTCAT & $6332-6351$ & 2095 \\
HN1 & TAGAACGGTCAGAGGAGCCA & $8407-8427$ & \\
\hline
\end{tabular}

the two viruses. An $\mathrm{R}$ value of $\mathrm{R}<0.5$ indicates a major antigenic difference between the two virus strains.

\section{Test of the protective efficacy of the LaSota strain against the variant strains}

To determine whether the vaccine strain LaSota with a low antibody level can fully protect against the variant strains, two strains, namely, JS-22-11-Ch (347E, 362G) and JS-14-12-Ch (347 K, 362A), were selected, and a locally produced, inactivated, oil-emulsion vaccine derived from the LaSota strain $\left(10^{9.3} \operatorname{EID}_{50}\right)$ was used in this study [25]. Twenty-five birds were immunized via the intramuscular route with a single dose $(0.1 \mathrm{ml})$ of the LaSota vaccine. Fifteen birds were not vaccinated and served as a control group. Three weeks post-vaccination, 20 vaccinated birds were chosen and divided into two groups of 10 . The birds in the La-JS22 group were challenged with $10^{6.0} \mathrm{EID}_{50} / 100 \mu \mathrm{l}$ JS-22-11-Ch $(n=10)$ via the eye drop and intranasal route $(\mathrm{ED} / \mathrm{IN}$ route; $100 \mu \mathrm{l})$, and those in the La-JS14 group were challenged with JS$14-12-\mathrm{Ch}(n=10)$ via same $(100 \mu \mathrm{l})$ route (ED/IN route). The unvaccinated birds were also challenged with JS-2211-Ch $(n=5)$ or JS-14-12-Ch $(n=5)$ as described above and were grouped in the Control-JS22 and Control-JS14 groups. Ten birds in the Control-La and Control-PBS groups (five LaSota-vaccinated birds and five unvaccinated birds) were challenged with the same volume $(100 \mu \mathrm{l})$ of PBS via the same route. All of the birds were monitored daily for overt clinical signs (depression, respiratory signs, diarrhea, etc.) and mortality. Tracheal and cloacal swabs were collected from birds at days 3, 5, and 7 post-challenge (pc) for virus isolation.

\section{Abbreviations}

CEF, chicken embryo fibroblasts; F, fusion protein; HI, hemagglutination inhibition; HN, hemagglutinin-neuraminidase; L, large polymerase protein; M, matrix protein; ND, Newcastle disease; NDV, Newcastle disease virus; NP, nucleocapsid protein; $P$, phosphoprotein; SPF, specific-pathogen-free

\section{Acknowledgements}

The authors would like to recognize Mrs. Liu Huimou, Ding Jihong and Gu kaihong for their significant contributions to the success of this study.

\section{Funding}

This work was supported by Chinese Special Fund for Agro-Scientific Research in the Public Interest (201303033, 201003012), the Earmarked Fund for Modern Agro-Industry Technology Research System (nycytx-41-G07), National Natural Science Foundation of China (31172338), and the Priority Academic Program Development of Jiangsu Higher Education Institutions (PAPD).

Availability of data and materials

All the data supporting the findings is contained within the manuscript.

\section{Authors' contributions}

$\mathrm{JZ}$ and SH carried out the study design, phylogenetic analysis, sequence alignment, and drafted the manuscript. HX contributed for RNA preparation and RT-PCR. JL, ZZ participated in the whole procedure of the virus isolation and pathogenicity tests. XW contributed to the design of the study and revision of the manuscript. XL conceived of the study, provided consultation and coordination, and helped to draft the manuscript. All authors read and approved the final manuscript.

\section{Competing interests}

The authors declare that they have no competing interests.

\section{Consent to publish}

Not applicable.

\section{Ethics approval and consent to participate}

Experimental protocols were approved and conducted by the Laboratory Animal Monitoring Committee of Jiangsu province of China. All experiments involving live viruses and animals were housed in negative-pressure isolators with HEPA filters. All samples were collected under the permission of the flock owners.

\section{Author details}

${ }^{1}$ Animal Infectious Disease Laboratory, College of Veterinary Medicine, Yangzhou University, 12 East Wenhui Road, Yangzhou, Jiangsu 225009, China. ${ }^{2}$ Jiangsu Co-innovation Center for Prevention and Control of Important Animal Infectious Diseases and Zoonoses, Yangzhou University, Yangzhou 225009, China. '3handong Binzhou Wohua Biological Engineering Co., Ltd, Binzhou 256600, China.

Received: 9 October 2015 Accepted: 7 June 2016

Published online: 16 June 2016

\section{References}

1. Aldous EW, Alexander DJ. Detection and differentiation of Newcastle disease virus (avian paramyxovirus type 1). Avian Pathol. 2001;30:117-28.

2. Mayo MA. A summary of taxonomic changes recently approved by ICTV. Arch Virol. 2002;147:1655-63.

3. Mayo MA. Virus taxonomy - Houston 2002. Arch Virol. 2002;147:1071-6.

4. Peeters BP, de Leeuw OS, Verstegen I, Koch G, Gielkens AL. Generation of a recombinant chimeric Newcastle disease virus vaccine that allows serological differentiation between vaccinated and infected animals. vaccine. 2001;19:1616-27.

5. Iorio RM, Syddall RJ, Sheehan JP, Bratt MA, Glickman RL, Riel AM. Neutralization map of the hemagglutinin-neuraminidase glycoprotein of Newcastle disease virus: domains recognized by monoclonal antibodies that prevent receptor recognition. J Virol. 1991;65:4999-5006. 
6. Cho SH, Kwon HJ, Kim TE, Kim JH, Yoo HS, Kim SJ. Variation of a newcastle disease virus hemagglutinin-neuraminidase linear epitope. J Clin Microbiol. 2008:46:1541-4.

7. Wu S, Wang W, Yao C, Wang X, Hu S, Cao J, Wu Y, Liu W, Liu X. Genetic diversity of Newcastle disease viruses isolated from domestic poultry species in Eastern China during 2005-2008. Arch Virol. 2010;156:253-61.

8. Cho SH, Kim SJ, Kwon HJ. Genomic sequence of an antigenic variant Newcastle disease virus isolated in Korea. Virus Genes. 2007;35:293-302.

9. Choi KS, Kye SJ, Kim JY, To TL, Nguyen DT, Lee YJ, Choi JG, Kang HM, Kim KI, Song BM, Lee HS. Molecular epidemiology of Newcastle disease viruses in Vietnam. Trop Anim Health Prod. 2014;46:271-7.

10. Kwon HJ, Cho SH, Ahn YJ, Seo SH, Choi KS, Kim SJ. Molecular epidemiology of Newcastle disease in Republic of Korea. Vet Microbiol. 2003;95:39-48.

11. Umali DV, Ito H, Suzuki T, Shirota K, Katoh H, Ito T. Molecular epidemiology of Newcastle disease virus isolates from vaccinated commercial poultry farms in non-epidemic areas of Japan. Virol J. 2013;10:330.

12. Zhang YY, Shao MY, Yu XH, Zhao J, Zhang GZ. Molecular characterization of chicken-derived genotype VIId Newcastle disease virus isolates in China during 2005-2012 reveals a new length in hemagglutinin-neuraminidase. Infect Genet Evol. 2014;21:359-66.

13. Rui Z, Juan P, Jingliang $S$, Jixun Z, Xiaoting W, Shouping Z, Xiaojiao L, Guozhong Z. Phylogenetic characterization of Newcastle disease virus isolated in the mainland of China during 2001-2009. Vet Microbiol. 2010; 141:246-57.

14. Zhang S, Wang X, Zhao C, Liu D, Hu Y, Zhao J, Zhang G. Phylogenetic and pathotypical analysis of two virulent Newcastle disease viruses isolated from domestic ducks in China. PLoS One. 2011;6, e25000.

15. Wang J, Liu H, Liu W, Zheng D, Zhao Y, Li Y, Wang Y, Ge S, Lv Y, Zuo Y, et al. Genomic Characterizations of Six Pigeon Paramyxovirus Type 1 Viruses Isolated from Live Bird Markets in China during 2011 to 2013. PLoS One. 2015;10, e0124261.

16. Ebrahimi MM, Shahsavandi S, Moazenijula G, Shamsara M. Phylogeny and evolution of Newcastle disease virus genotypes isolated in Asia during 2008-2011. Virus Genes. 2012;45:63-8.

17. Hu S, Wang T, Liu Y, Meng C, Wang X, Wu Y, Liu X. Identification of a variable epitope on the Newcastle disease virus hemagglutininneuraminidase protein. Vet Microbiol. 2010:140:92-7.

18. Cho SH, Kwon HJ, Kim TE, Kim JH, Yoo HS, Park MH, Park YH, Kim SJ. Characterization of a recombinant Newcastle disease virus vaccine strain. Clin Vaccine Immunol. 2008;15:1572-9.

19. Qin ZM, Tan LT, Xu HY, Ma BC, Wang YL, Yuan XY, Liu WJ. Pathotypical characterization and molecular epidemiology of Newcastle disease virus isolates from different hosts in China from 1996 to 2005. J Clin Microbiol. 2008:46:601-11.

20. Harper DR. Anovel plaque assaysystem for paramyxoviruses. J Virol Methods. 1989;25.

21. Liu XF, Wan HQ, Ni XX, Wu YT, Liu WB. Pathotypical and genotypical characterization of strains of Newcastle disease virus isolated from outbreaks in chicken and goose flocks in some regions of China during 1985-2001. Arch Virol. 2003:148:1387-403.

22. Tamura K, Dudley J, Nei M, Kumar S. MEGA4: Molecular Evolutionary Genetics Analysis (MEGA) software version 4.0. Mol Biol Evol. 2007;24:1596-9.

23. Li ZJ, Li Y, Chang S, Ding Z, Mu LZ, Cong YL. Antigenic variation between Newcastle disease viruses of goose and chicken origin. Arch Virol. 2010;155: 499-505.

24. Archetti I, Horsfall Jr FL. Persistent antigenic variation of influenza A viruses after incomplete neutralization in ovo with heterologous immune serum. J Exp Med. 1950;92:441-62.

25. Hu Z, Hu S, Meng C, Wang X, Zhu J, Liu X. Generation of a genotype VII Newcastle disease virus vaccine candidate with high yield in embryonated chicken eggs. Avian Dis. 2011:55:391-7.

\section{Submit your next manuscript to BioMed Central and we will help you at every step:}

- We accept pre-submission inquiries

- Our selector tool helps you to find the most relevant journal

- We provide round the clock customer support

- Convenient online submission

- Thorough peer review

- Inclusion in PubMed and all major indexing services

- Maximum visibility for your research

Submit your manuscript at www.biomedcentral.com/submit
Biomed Central 